\title{
Langerhans Cell Histiocytosis with Extensive Spinal and Thyroid Gland Involvement Presenting with Quadriparesis: An Unusual Case in an Adult Patient
}

\author{
Mohd Ariff S, MBBS, Joehaimey J*, M MED Orth, Ahmad Sabri O*, M MED Orth \\ Zulmi $\mathbf{W} * *$, MS Orth \\ Department of Orthopaedics, Traumatology and Rehabilitation, \\ International Islamic University Malaysia, Kuantan, Malaysia \\ *Department of Orthopaedics, Hospital Raja Perempuan Zainab II (HRPZII), Kota Bharu, Malaysia \\ **Department of Orthopaedics, School of Medical Sciences, Universiti Sains Malaysia, Kota Bharu, Malaysia
}

\begin{abstract}
Langerhans cell histiocytosis of the spine frequently presents as a solitary lesion and rarely results in neurological deficit. Involvement of thyroid tissue is also rare, even in multifocal disease presentations. We present an unusual variant of Langerhans cell histiocytosis in a 37-year-old man presenting with profound quadriparesis and incidental thyroid involvement. MR imaging and skeletal radiographs revealed widespread involvement of the spine. The patient underwent surgical resection of the tumour and stabilization of the spine. Diagnosis was confirmed by histological and immunohistochemistry analysis. Langerhans cell histiocytosis should be included in the differential diagnosis of radiolucent lesions of the spine in adults.
\end{abstract}

Key Words:

Langerhans Cell Histiocytosis, Spine Tumours, Thyroid Mass, Cord Compression With Quadriparesis, Adult

\section{INTRODUCTION}

The designation of Langerhans cell histiocytosis (LCH) replaced the previous nomenclature for the group of disorders termed histiocytosis $\mathrm{X}$, which included eosinophilic granuloma (EG), Hand-Schüller-Christian Disease, and Letterer-Siwe Disease. These conditions vary greatly in presentation and outcome, but share similar recognition of clonal proliferation and accumulation of a specific histiocyte, the Langerhans cell. LCH is primarily a disease of children, and is usually presents before the age of 20 years $^{1}$. Vertebral involvement is relatively common, but widespread spinal LCH is rarely reported'. Furthermore, involvement of the spinal cord and roots remains a rare occurrence $e^{2}$. Similarly, involvement of the thyroid gland (in either children or adults, particularly in adults), even in the setting of multifocal disease, is rare $^{3}$ In this report, we present presentation of multifocal $\mathrm{LCH}$ of the spine, causing cord compression and quadriparesis, with an incidental finding of unifocal EG of the thyroid gland.

\section{CASE REPORT}

A 37-year-old male presented with a 5-month history of progressive back pain associated with paraparesis. He was first, evaluated at a primary medical centre, and was treated with analgesics. The patient was also noted to have an asymptomatic $3 \times 3 \mathrm{~cm}$ thyroid mass. Fine needle aspiration of the mass was performed before he was referred to our centre. The patient reported spontaneous onset of mid-thoracic back pain, which was constant and prickly in nature and intensity increased at night. There was no history of spinal trauma. He also reported generalized weakness, weight loss, and lowgrade fever. There was no loss of sphincter control, but he needed support to walk. He denied any contact history with tuberculosis. Family and medical history were unremarkable. Physical examination revealed tenderness elicited over the spinous processes of T6 and T7. Sensation was reduced over the right side at the level of $\mathrm{T} 7$ and $\mathrm{T} 8$. Voluntary power in all muscle groups was fairly normal. Perirectal examination revealed no abnormal findings. No lymphadenopathy was found. Other systemic reviews were unremarkable.

Chest radiographs and most laboratory tests (including full blood counts and differentials, serum electrolytes, liver and thyroid function tests) were in the normal range. Exceptions included a slight elevation of total white cells and an erythrocyte sedimentation rate of $70 \mathrm{~mm} / \mathrm{h}$. The Mantoux test was positive. Plain radiographs of the thoracic, lumbar and sacral spine showed disruption of pedicles at T6/T7 with osteolytic changes in T4-T6, L3-L4, and S1, as well as vertebral collapse of L4. Magnetic resonance imaging revealed multiple vertebral and localized right ilium destruction. In view of the patient's age, as well as laboratory

Corresponding Author: Mohd Ariff Sharifudin, Department of Orthopaedics, Traumatology and Rehabilitation, Kulliyyah of Medicine, International Islamic University Malaysia, Jalan Hospital, 25100 Kuantan, Pahang, Malaysia

Email: ariffs@iium.edu.my 


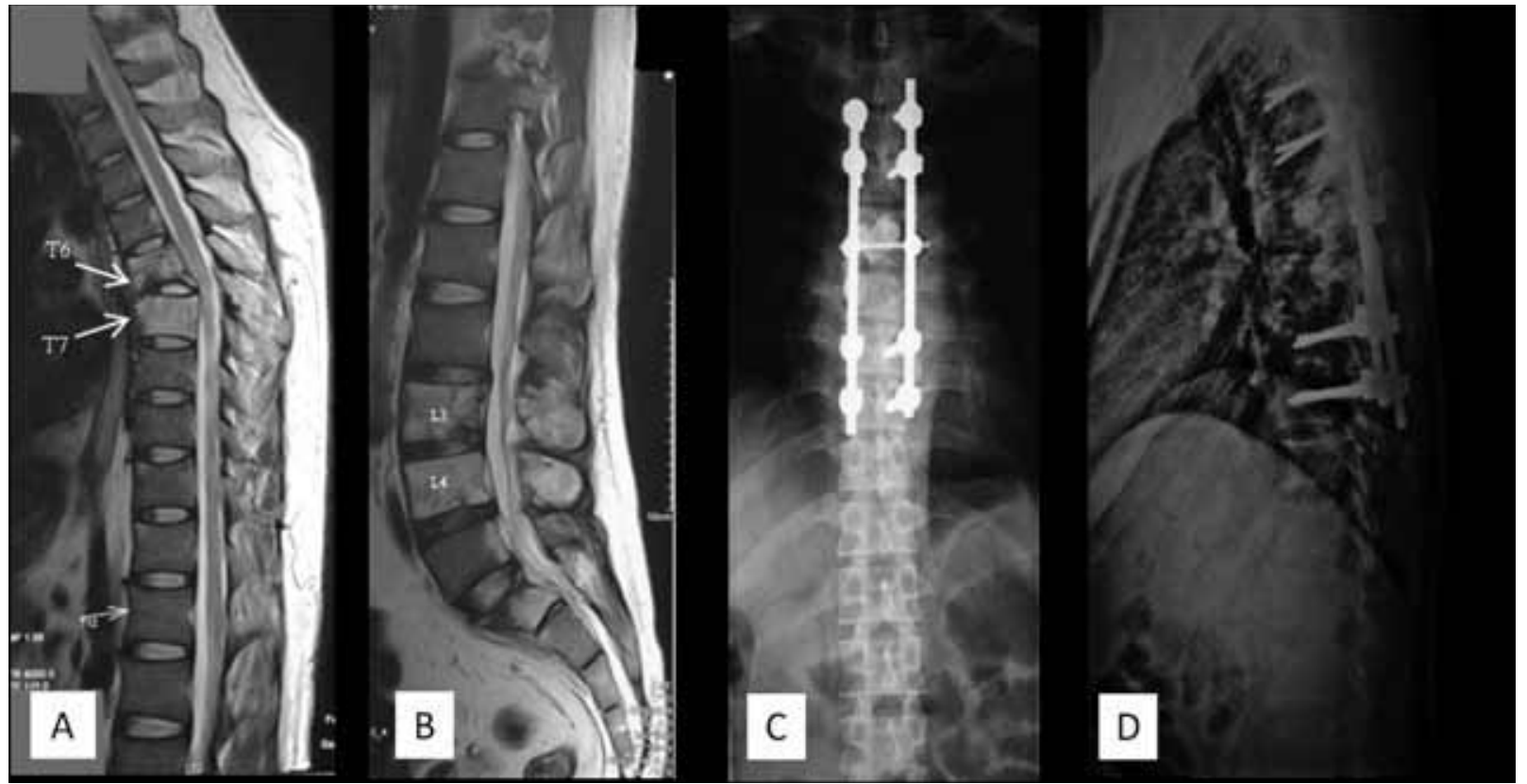

Fig. 1: (A) MR imaging (T1-weighted) of the thoracic spine. Note the disruption of T6 and T7. (B) Lumbosacral spine on MR imaging (T1-weighted) with osteolytic changes on L3 and L4. (C and D) Post-operative plain radiographs of the thoracic spine.

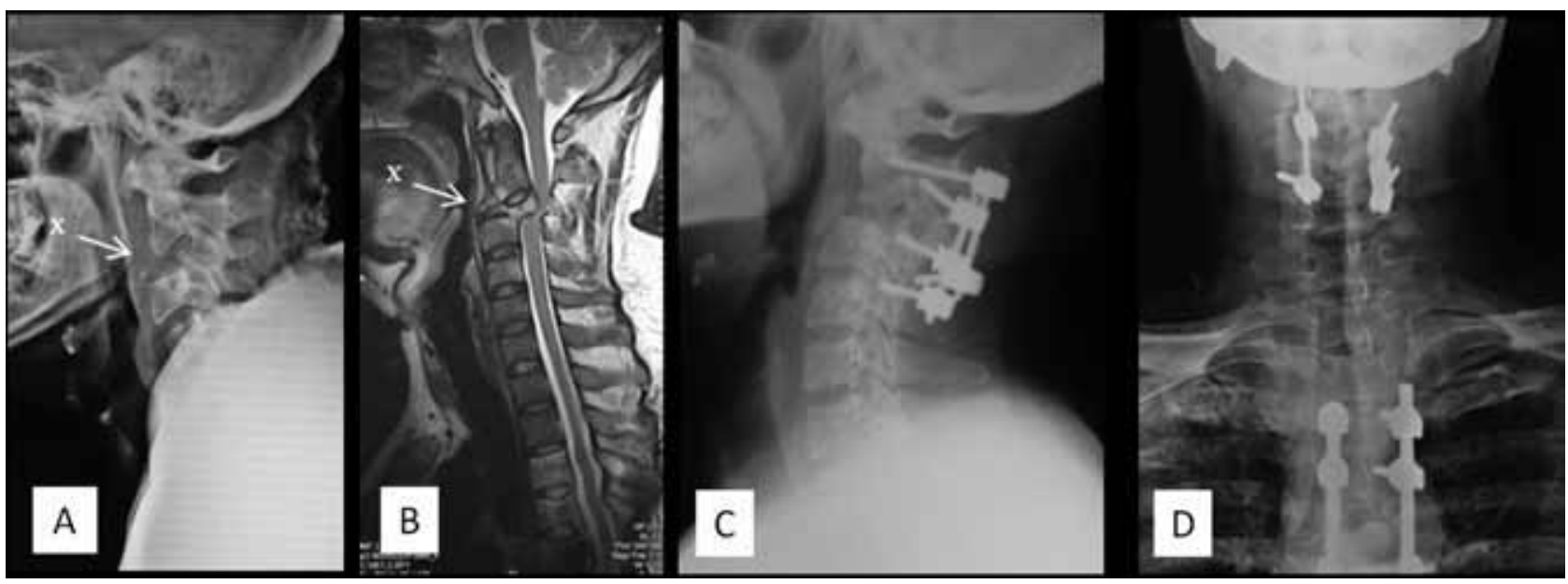

Fig. 2: (A) Plain radiograph (lateral view) and (B) MR imaging (T1-weighted) of the cervical spine, revealing subluxation of $C 3 / C 4$ (X). Post-operative plain radiographs, (C) lateral view and (D) anteroposterior view.

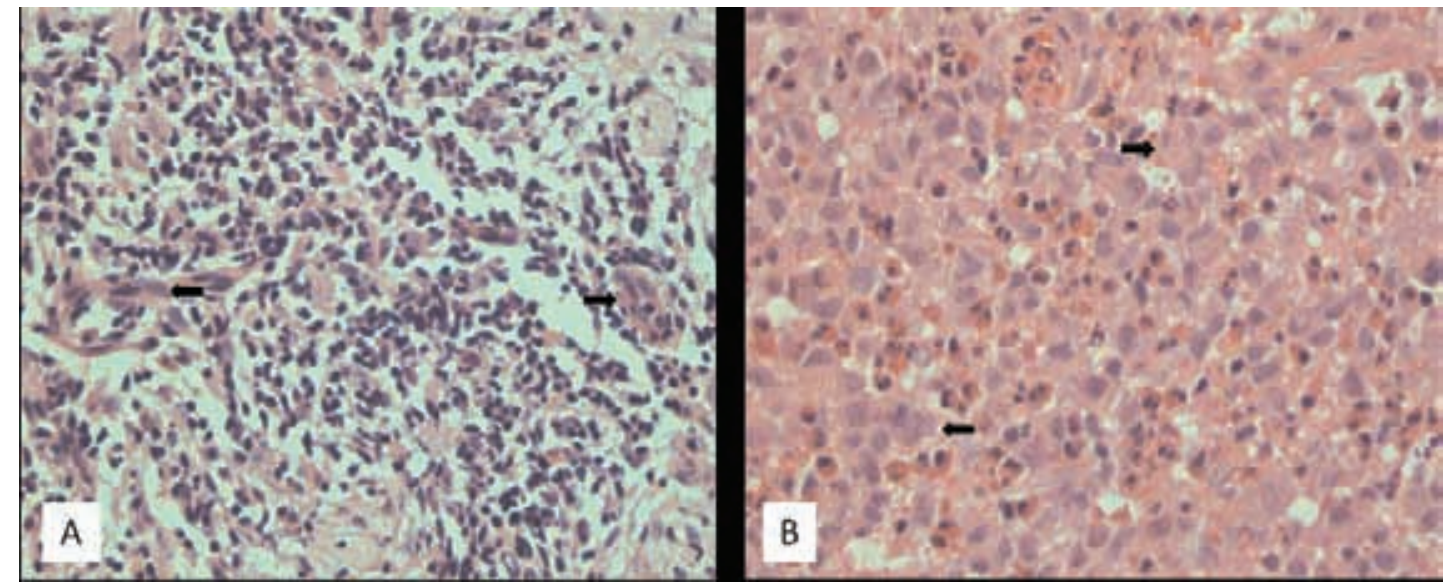

Fig. 3: Histological section from (A) T7 vertebral body, and (B) body of C3 vertebra, demonstrating large pale-staining histiocytes (arrows) with eccentric nuclei and nuclear grooves. There is also a proliferation of mixed inflammatory cells characterized by cells with dark-staining nuclei, consistent with lymphocytes, eosinophils and plasma cells (haematoxylin and eosin, x40). 
and examination results, the provisional diagnosis was spinal tuberculosis, but metastasis and lymphoma were also considered.

The patient underwent a posterior decompression procedure with curettage and posterior instrumentation and bone biopsy of the T6 and T7 vertebrae. Intraoperatively, there was abnormal soft, grey extrathecal tissue with destruction and fragile T6 and T7 vertebral bodies. Histopathologic examination revealed chronic inflammation with no evidence of malignancy or infection. Postoperatively the patient was empirically prescribed first line anti-tuberculosis drug regime and responded well. At routine follow up appointments, the patient was ambulating well and able to independently perform daily activities.

Unfortunately, 18 months following the surgery, the patient presented with quadriparesis and inability to micturate. There was tenderness over the cervical spinous processes. Voluntary power of bilateral upper and lower limbs was at a Medical Research Council (MRC) score of 3, with reduced sensation from level C6 downward. Other cranial nerves were intact. Signs of upper motor neuron lesion were also observed. Repeated MR imaging revealed subluxation of $\mathrm{C} 3 / \mathrm{C} 4$, with cord compression. There was also destruction of the $\mathrm{C} 3$ vertebral body with paravertebral soft tissue swelling. The patient underwent emergency open reduction, decompression, curettage and interbody fusion of $\mathrm{C} 2$ to $\mathrm{C} 4$ with anterior bone grafting and posterior instrumentation. New bone and soft tissue biopsies were obtained and evaluated alongside the previous biopsy samples. Histopathologic evaluation revealed mixed cellular infiltrate made up of predominantly lymphocytes, neutrophils, eosinophils and histiocytes. Histiocytic elements presented as typical indented nuclei with abundant cytoplasm. Immunohistochemistry analysis revealed high levels of cytoplasmic content including S-100 protein and those classified as Langerhans cells. The diagnosis of Langerhans cell histiocytosis was made.

Following the second surgery, the patient's motor function gradually improved; this was followed by improved sensory function. Examination revealed complete neurological recovery three months after the second surgery. Histopathologic examination and immunohistochemistry analysis of the thyroid tissue obtained during the earlier phase was also consistent with LCH. Complete skeletal survey and CT scan of the brain were performed to exclude other bony lesions and pituitary gland involvement. In view of the multifocal lesions of the disease, we recommended chemotherapy, but the patient refused this. The patient has continued with follow-up for the past 24 months. Thyroid function remains asymptomatic with no further enlargement seen. There was no worsening of spinal lesions and no new lesions discovered. He went back to his pre-disease daily activities without any signs of recurrence.

\section{DISCUSSION}

$\mathrm{LCH}$ presents a diagnostic dilemma among adult patients; there are large numbers of relatively more common lesions with a similar appearance; thus there is low index of suspicion among practitioners for $\mathrm{LCH}$. The presence of constitutional symptoms, as in our patient, can also complicate the diagnosis.

The most common site in the axial skeleton is the skull ${ }^{1,4}$. Vertebral involvement is most common in paediatric patients $^{1,4}$ and presentation is generally as a solitary lesion'. In adults, the condition presents as a thoracic lesion followed by lumbar and cervical spine presentations. Spinal lesions occur mainly in the vertebral body without soft tissue or posterior arch extension ${ }^{1-2,45}$. Typical presentation includes dull back pain without neurological involvement. In the current case, there was involvement of the paravertebral structures as well as $\mathrm{C} 3 / \mathrm{C} 4$ subluxation, which lead to quadriparesis.

There exists a lack of published reports about extensive spinal LCH. The issue of management protocols for extensive spinal LCH remains controversial and no standard principles of treatment are available to date. Goals of treatment include spinal stability and preservation of neurological function. As the disease is self-limiting, surgical intervention is not always recommended. Chemotherapy is recommended for multiple lesions ${ }^{2,5}$, but the benefits of radiotherapy are still under debate ${ }^{1-2,5}$. Simultaneous involvement of the thyroid, pituitary, and parathyroid glands has been reported, sometimes in association with diabetes insipidus ${ }^{3}$. Hence, such conditions should be ruled out during evaluation.

This report is presented to emphasize the importance of:

(1) including LCH in the differential diagnosis process for radiolucent lesions of the spine in adults;

(2) the possibility of cord compression although this has been reported rarely; and

(3) variability in clinical presentation and histology of $\mathrm{LCH}$, and as such requires higher degree of suspicion. 


\section{REFERENCES}

1. Bertram C, Madert J, Eggers C. Eosinophilic granuloma of the cervical spine. Spine. 2002; 27(13): 1408-13.

2. Jiang L, Liu XG, Zhong WQ, Ma QJ, Wei F, Yuan HS, et al. Langerhans cell histiocytosis with multiple spinal involvement. Eur Spine J. 2010 May 22. DOI 10.1007/s00586-010-1390-1

3. Yap WM, Chuah KL, Tan PH. Langerhans cell histiocytosis involving the thyroid and parathyroid glands. Mod Pathol 2001; 14(2): 111-5.

4. Islinger RB, Kuklo TR, Owens BD, Horan PJ, Choma TJ, Murphey MD, et al. Langerhans cell histiocytosis in patients older than 21 years. Clin Orthop Relat Res. 2000; 10(379): 231-5.

5. Huang W, Yang X, Cao D, Xiao J, Yang M, Feng D, et al. Eosinophilic granuloma of spine in adults: a report of 30 cases and outcome. Acta Neurochir (Wien). 2010; 152(7): 1129-37. 